\title{
ASYMPTOTIC TOEPLITZ OPERATORS
}

\author{
BY
}

JOSÉ BARRIA AND P. R. HALMOS ${ }^{1}$

\begin{abstract}
An asymptotic Toeplitz is an operator $T$ such the sequence $\left\{U^{* n} T U^{n}\right\}$ is strongly convergent, where $U$ is the unilateral shift. Every element of the norm-closed algebra generated by all Toeplitz and Hankel opertors together is an asymptotic Toeplitz operator. The authors study the relations among this Hankel algebra, the classical Toeplitz algebra, the set of all asymptotic Toeplitz operators, and the essential commutant of the unilateral shift. They offer several examples of operators in some of these classes but not in others, and they raise several open questions.
\end{abstract}

What is the essential commutant of the unilateral shift? The experts are convinced that, whatever it is, it is huge. The purpose of this paper is to call attention to an asymptotic property of some operators, use that property to show that certain concrete operators that do not belong to the Toeplitz algebra do belong to the essential commutant of the shift, discuss some related examples, and pose a few unsolved problems.

The Toeplitz and Hankel algebras. The underlying Hilbert space is $\mathbf{H}^{2}$ of the unit circle. The unilateral shift $U$ is defined on $\mathbf{H}^{2}$ by $U f(z)=z f(z)$. The essential commutant of $U$ is, by definition, the set $\mathbf{E}$ of all those operators $T$ on $\mathbf{H}^{2}$ for which $U T-T U \in \mathbf{K}$ (where $\mathbf{K}$ is the ideal of all compact operators on $\mathbf{H}^{2}$ ).

Since $U$ is essentially unitary (i.e., both $U^{*} U$ and $U U^{*}$ are congruent to $1 \bmod \mathbf{K}$ ), it follows that $T \in \mathbf{E}$ if and only if $U^{*} T U-T \in \mathbf{K}$. This reformulation of the definition of $\mathbf{E}$ is convenient in matrix calculations. (For operators on $\mathbf{H}^{2}$, all matrices in the sequel will be formed with respect to the basis $\left\{e_{0}, e_{1}, e_{2}, \ldots\right\}$ defined by $\left.e_{n}(z)=z^{n}, n=0,1,2, \ldots\right)$ Since, in terms of the Kronecker delta, the matrix of $U$ is $\left(\delta_{i, j+1}\right)$, the matrix of a product $T U$ is obtained from the matrix of $T$ by erasing the first column, and the matrix of $U^{*} T$ is obtained from that of $T$ by erasing the first row. (Caution: "erase" means literally what it says; it does not mean "replace by 0 's".) The matrix of $U^{*} T U$, therefore, is obtained from that of $T$ by "moving one step to the southeast"; to say that $T \in \mathbf{E}$ is the same as to say that, $\bmod \mathbf{K}$, the matrix is not changed by the move.

The essential commutant of every operator is a norm-closed algebra. Since $\mathbf{E}$ contains every Toeplitz operator (recall a possible definition: $U^{*} T U=T$ ), it follows that the Toeplitz algebra (the norm-closed algebra $\mathbf{T}$ generated by the set of all

Received by the editors June 1, 1981.

1980 Mathematics Subject Classification. Primary 47B35, 47B37.

'Research supported in part by a grant from the National Science Foundation. 
Toeplitz operators) is included in E. Question, with a not immediately obvious answer: is $\mathbf{E}$ equal to $\mathbf{T}$ ? The experts' conviction ( $\mathbf{E}$ is huge) means, among other things, that the answer is no; some concrete examples of operators in $\mathbf{E}$ but not in $\mathbf{T}$ will become visible presently. (The most important earlier work on a closely related problem is [2].)

In view of the role that $\mathbf{K}$ plays in the definition of essential commutativity, the relation $\mathbf{K} \subset \mathbf{E}$ is even more obvious than the relation $\mathbf{T} \subset \mathbf{E}$. It is not only obvious: it contains no new information. Reason: $\mathbf{K} \subset \mathbf{T}$. This inclusion can be inferred from a sophisticated fact about irreducible $C^{*}$-algebras [3,p. 141], or can be proved directly. [Note that since $U$ is essentially unitary, it follows that $\mathbf{E}$ is closed under the formation of adjoints and is therefore a $C^{*}$-algebra. Since $U$ is irreducible and $U \in \mathbf{E}$, it follows that $\mathbf{E}$ is irreducible.] Here is an elementary direct proof. Since $U \in \mathbf{T}$, therefore $E=1-U U^{*} \in \mathbf{T}$; the operator $E$ is, in fact, the projection $e_{0} \otimes e_{0}$ of rank 1. For arbitrary operators $S$ and $T$, the product $S\left(e_{0} \otimes e_{0}\right) T$ is equal to $\left(S e_{0}\right) \otimes\left(T^{*} e_{0}\right)$; it follows that if $S$ and $T$ are in $\mathbf{T}$, then so is $\left(S e_{0}\right) \otimes\left(T^{*} e_{0}\right)$. If, in particular, $p$ and $q$ are arbitrary polynomials, and if $S=p(U)$ and $T=q(U)^{*}$, then $\left(p(U) e_{0}\right) \otimes\left(q(U) e_{0}\right) \in \mathbf{T}$. Since the set of all vectors obtained by applying a polynomial in $U$ to $e_{0}$ is dense in $\mathbf{H}^{2}$, it follows that every operator of rank 1 is in $\mathbf{T}$, and so therefore is every compact operator.

If $\varphi \in \mathbf{L}^{\infty}$ of the unit circle, write $M_{\varphi}$ for the multiplication operator defined on $\mathbf{L}^{2}$ by $M_{\varphi} f=\varphi f$, and $T_{\varphi}$ for the compression defined on $\mathbf{H}^{2}$ by $T_{\varphi} f=P M_{\varphi} f$ (where $P$ is the projection from $\mathbf{L}^{2}$ onto $\mathbf{H}^{2}$ ). The compression $T_{\varphi}$ is a Toeplitz operator, and every Toeplitz operator is obtained this way. If $M_{\varphi}$ is expressed as an operator matrix with respect to the decomposition $\mathbf{L}^{2}=\mathbf{H}^{2^{\perp}} \oplus \mathbf{H}^{2}$, the result is of the form

$$
M_{\varphi}=\left(\begin{array}{cc}
T_{\tilde{\varphi}} & H_{\varphi} \\
H_{\tilde{\varphi}} & T_{\varphi}
\end{array}\right),
$$

where $\tilde{\varphi}(z)=\varphi\left(z^{*}\right)$, the diagonal entries are Toeplitz operators, and the others are Hankel operators. (The latter can be defined by this remark; alternatively a Hankel operator $H$ is one for which $U^{*} H=H U$.) If $\varphi$ and $\psi$ are in $\mathrm{L}^{\infty}$, then $M_{\varphi \psi}=M_{\varphi} M_{\psi}$, and therefore (mulitply matrices and compare lower right corners)

$$
T_{\varphi \psi}=T_{\varphi} T_{\psi}+H_{\tilde{\varphi}} H_{\psi} .
$$

What is most important about this equation is that the product of two Toeplitz operators differs from a Toeplitz operator by the product of the two Hankel operators, and every product of two Hankel operators arises in this way. A related formula with a related proof (compare upper right corners) can also be useful:

$$
H_{\varphi \psi}=T_{\tilde{\varphi}} H_{\psi}+H_{\varphi} T_{\psi} \text {. }
$$

Hankel operators are an essential part of Toeplitz theory. An effective way to welcome them is to consider the Hankel algebra (the norm-closed algebra $\mathbf{T}^{+}$ generated by all Toeplitz operators and all Hankel operators together).

Convergence. It is natural to define an asymptotic Toeplitz operator as an operator $T$ such that the sequence $\left\{U^{* n} T U^{n}\right\}$ is strongly convergent. The limit is clearly a 
Toeplitz operator, and hence of the form $T_{\varphi}$ for some $\varphi$ in $\mathbf{L}^{\infty}$. The function $\varphi$ will be called the symbol of $T$ and will be denoted by $\sigma(T)$. The simplest examples are the Toeplitz operators; the next simplest the Hankel operators.

(3) LEMMA. If $H$ is a Hankel operator, then $H U^{n} \rightarrow 0$ (strong).

Proof. From the matrix point of view the statement is almost obvious: the matrix of $H U^{n}$ is obtained from that of $H$ by erasing the first $n$ columns. [Note that each entry occurs in a Hankel matrix only a finite number of times.] Alternatively, $H U^{n}=U^{* n} H$, and $U^{* n} \rightarrow 0$ (strong).

(4) THEOREM. Every element of the Hankel algebra is an asymptotic Toeplitz operator.

Proof. The main step is to show that if $\varphi_{1}, \ldots, \varphi_{k}$ are in $\mathbf{L}^{\infty}$, if $T=T_{\varphi_{1}} \cdots T_{\varphi_{k}}$, and if $\varphi=\varphi_{1} \cdots \varphi_{k}$, then $U^{* n} T U^{n} \rightarrow T_{\varphi}$ (strong). The argument is based on a telescoping sum:

$$
\begin{aligned}
T_{\varphi_{1}} \cdots T_{\varphi_{k}}-T_{\varphi_{1} \cdots \varphi_{k}}= & T_{\varphi_{1}} T_{\varphi_{2} \cdots \varphi_{k}}-T_{\varphi_{1}\left(\varphi_{2} \cdots \varphi_{k}\right)} \\
& +T_{\varphi_{1}}\left(T_{\varphi_{2}} T_{\varphi_{3} \cdots \varphi_{k}}-T_{\varphi_{2}\left(\varphi_{3} \cdots \varphi_{k}\right)}\right) \\
& +T_{\varphi_{1}} T_{\varphi_{2}}\left(T_{\varphi_{3}} T_{\varphi_{4} \cdots \varphi_{k}}-T_{\varphi_{3}\left(\varphi_{4} \cdots \varphi_{k}\right)}\right) \\
& +\cdots \\
& +T_{\varphi_{1}} T_{\varphi_{2}} \cdots T_{\varphi_{k-2}}\left(T_{\varphi_{k-1}} T_{\varphi_{k}}-T_{\varphi_{k-1} \varphi_{k}}\right)
\end{aligned}
$$

In view of this, equation (1) implies that

$$
T-T_{\varphi}=H H+T H H+T T H H+\cdots+T T \cdots T H H,
$$

where each $T$ on the right side indicates a Toeplitz operator and each $H$ a Hankel operator; since the actual subscripts are useless, they are omitted. Multiply by $U^{* n}$ on the left and $U^{n}$ on the right; since $T_{\varphi}$ is invariant under that operation, and since (by Lemma (3)) the right side converges strongly to 0 as $n \rightarrow \infty$, the main step is complete.

Consider next a finite product all whose factors are either Toeplitz or Hankel operators, with at least one Hankel factor present. If the rightmost factor is a Hankel operator, the asserted strong convergence (to 0 ) follows from Lemma (3). In the remaining cases, the first Hankel factor from the right occurs in a context $H T$, where, as before, the symbols $H$ and $T$ indicate generic Hankel and Toeplitz operators respectively. In such a case, use (2) to replace $H T$ by $H-T H$ (subscripts still omitted), and thus replace the given operator by two others, in each of which the rightmost Hankel factor is one step nearer to the right end; the desired convergence now follows by induction.

The rest is easy. Let $\mathbf{T}_{0}^{+}$be the (unclosed) algebra consisting of all finite sums of finite products of Toeplitz and Hankel operators. If $T \in \mathbf{T}_{0}^{+}$, convergence follows from the strong continuity of operator addition. For norm limits of operators in $\mathbf{T}_{0}^{+}$, convergence follows from the standard techniques of " $\frac{\varepsilon}{3}$ " analysis. 
(5) Corollary. The restriction of the symbol map o to the Hankel algebra is a contractive *-homomorphism from $\mathbf{T}^{+}$onto $\mathbf{L}^{\infty}$.

Proof. That $\sigma$ is a contraction is immediate from the strong lower semicontinuity of norm: if $U^{* n} T U^{n} \rightarrow T_{\varphi}$ (strong), then

$$
\|\sigma(T)\|_{\infty}=\|\varphi\|_{\infty}=\left\|T_{\varphi}\right\| \leqslant \liminf _{n}\left\|U^{* n} T U^{n}\right\| \leqslant\|T\| .
$$

That $\sigma$ preserves sums and products in $\mathbf{T}_{0}^{+}$follows from the main step in the preceding proof; that it preserves sums and products for all operators in the Hankel algebra follows from the (norm) continuity of operator addition and multiplication and the (just proved) continuity of $\sigma$. As for adjoints, there seems to be a difficulty; adjunction is not strongly continuous. Suppose, however, that $T \in \mathbf{T}^{+}$and $U^{* n} T U^{n}$ $\rightarrow T_{\varphi}$ (strong); the weak continuity of adjunction implies that $U^{* n} T^{*} U^{n} \rightarrow T_{\varphi}^{*}=T_{\varphi^{*}}$ (weak). Since $T^{*} \in \mathbf{T}^{+}$, the sequence $\left\{U^{* n} T^{*} U^{n}\right\}$ converges strongly to something, say $T_{\psi}$. Conclusion: $T_{\psi}=T_{\varphi^{*}}$, and therefore $\sigma\left(T^{*}\right)=\sigma(T)^{*}$.

The symbol map was originally defined for Toeplitz operators only; the existence of a homomorphic extension to the entire Hankel algebra yields a slight improvement of a curious result of Douglas [4, p. 9].

(6) COROLlaRY. If a finite sum of finite products of Toeplitz or Hankel operators is compact, then the corresponding finite sum of finite products of their symbols is zero almost everywhere.

Proof. If $K$ is compact, then $K U^{n} e_{j}=K e_{j+n} \rightarrow 0$ as $n \rightarrow \infty$ and therefore $\sigma(K)=0$; in other words $\mathbf{K} \subset \operatorname{ker} \boldsymbol{\sigma}$.

An important part of Toeplitz theory concerns the commutator ideal $\mathbf{Q}$ of the algebra $\mathbf{T}$ (see [3, p. 181]); the following characterization of $\mathbf{Q}$ might be useful.

(7) TheOREM. An operator $T$ in the Toeplitz algebra $\mathbf{T}$ belongs to the commutator ideal $\mathbf{Q}$ of $\mathbf{T}$ if and only if $U^{* n} T U^{n} \rightarrow 0$ (strong); equivalently $\mathbf{Q}=\operatorname{ker} \sigma$.

Proof. Suppose first that $\varphi_{1}, \ldots, \varphi_{k}$ are in $\mathbf{L}^{\infty}, T=T_{\varphi_{1}} \cdots T_{\varphi_{k}}$, and $\psi=\varphi_{1} \cdots \varphi_{k}$. Assertion: $T-T_{\psi} \in \mathbf{Q}$. The proof is induction on $k$. For $k=1$, the assertion is trivial. To pass from $k-1$ to $k$ assume, temporarily, that $\varphi_{k}=\alpha^{*} \beta$, where $\alpha$ and $\beta$ are in $\mathbf{H}^{\infty}$; then

$$
\begin{aligned}
T- & T_{\psi}=T_{\varphi_{1}} \cdots T_{\varphi_{k-1}} T_{\alpha^{*} \beta}-T_{\varphi_{1} \cdots \varphi_{k-1} \alpha^{*} \beta} \\
& =T_{\varphi_{1}} \cdots T_{\varphi_{k-1}} T_{\alpha^{*}} T_{\beta}-T_{\alpha^{*}} T_{\varphi_{1} \cdots \varphi_{k-1}} T_{\beta} \\
& =\left(T_{\varphi_{1}} \cdots T_{\varphi_{k-1}} T_{\alpha^{*}}-T_{\alpha^{*}} T_{\varphi_{1} \cdots \varphi_{k-1}}\right) T_{\beta} \\
& =\left(\left[T_{\varphi_{1}} \cdots T_{\varphi_{k-1}} T_{\alpha^{*}}-T_{\alpha^{*}} T_{\varphi_{1}} \cdots T_{\varphi_{k-1}}\right]+\left[T_{\alpha^{*}} T_{\varphi_{1}} \cdots T_{\varphi_{k-1}}-T_{\alpha^{*}} T_{\varphi_{1} \cdots \varphi_{k-1}}\right]\right) T_{\beta} .
\end{aligned}
$$

The first square bracket is a commutator, and therefore belongs to $\mathbf{Q}$. The second square bracket is $T_{\alpha^{*}}$ times an operator of the same form as $T-T_{\psi}$ except with $k-1$ instead of $k$, and, consequently, (by the induction hypothesis) it too belongs 
to $\mathbf{Q}$. At this point it seems necessary to use a relatively deep tool, namely the approximation theorem [3, p. 163] according to which functions of the form $\alpha^{*} \beta$ are dense in $\mathbf{L}^{\infty}$. With the use of that theorem the proof of the assertion is obviously complete; if $T-T_{\psi} \in \mathbf{Q}$ whenever $\varphi_{k}=\alpha^{*} \beta$, then $T-T_{\psi} \in \mathbf{Q}$ for all $\varphi_{k}$.

The preceding paragraph implies that if $T$ belongs to the (unclosed) algebra $\mathbf{T}_{0}$ consisting of all finite sums of finite products of Toeplitz operators, and if $\psi=\sigma(T)$, then $T-T_{\psi} \in \mathbf{Q}$. Indeed, suppose that $T=T_{1}+\cdots+T_{m}$, where each $T_{j}$ is a finite product of Toeplitz operators. It follows that $\psi=\psi_{1}+\cdots+\psi_{m}$, where $\psi_{j}=\sigma\left(T_{j}\right)$, $j=1, \ldots, m$, and hence that $T-T_{\psi}=\left(T_{1}-T_{\psi_{1}}\right)+\cdots+\left(T_{m}-T_{\psi_{m}}\right) \in \mathbf{Q}$.

Suppose now that $T$ is an arbitrary operator in $T$ with $\sigma(T)=0$. Let $\left\{T_{n}\right\}$ be a sequence, each term of which is an operator in $\mathbf{T}_{0}$, such that $T_{n} \rightarrow T$ (norm). If $\psi_{n}=\sigma\left(T_{n}\right)$, then $\psi_{n} \rightarrow 0$ in $\mathbf{L}^{\infty}$ (because $\sigma(T)=0$ ), and therefore $T_{n}-T_{\psi_{n}} \rightarrow T$ (norm). Since $T_{n}-T_{\psi_{n}} \in \mathbf{Q}$ for each $n$ (by the preceding paragraph), it follows that $T \in \mathbf{Q}$.

What was proved so far was that $\operatorname{ker} \sigma \subset \mathbf{Q}$. Since $\mathbf{T} / \operatorname{ker} \sigma$ is commutative, the reverse inclusion is trivial.

Examples. The condition $U^{*} T U-T \in \mathbf{K}$ is (necessary and) sufficient for $T \in \mathbf{E}$; the condtion that the sequence $\left\{U^{* n} T U^{n}\right\}$ be strongly convergent is necessary for $T \in \mathbf{T}$. Are these conditions sharp enough to distinguish between $\mathbf{E}$ and $\mathbf{T}$ ?

(8) Example. The Hankel operator $H$ whose matrix is $(1 /(i+j+1)), i, j=$ $0,1,2, \ldots$, (usually known as the Hilbert matrix) is a famous one; it is quite easy to see that it belongs to $\mathbf{E}$. Indeed, the matrix of $U^{*} H U$ is $(1 /(i+j+3))$; the difference $U^{*} H U-H$ has matrix

$$
\left(\frac{-2}{(i+j+1)(i+j+3)}\right) \text {. }
$$

Elementary analysis shows that the sum of the squares of all the entries in this difference is finite; in other words, $U^{*} H U-H$ is a Hilbert-Schmidt operator. Conclusion: $U^{*} H U-H \in \mathbf{K}$, so that $H \in \mathbf{E}$.

Is $H$ an asymptotic Toeplitz operator? The answer is yes, and the proof is easy. The necessary convergence condition is satisfied, and, for all that is visible at this stage, it could be that $H \in \mathrm{T}$.

The fact is that $H$ does belong to the Toeplitz algebra; the proof goes as follows. Since $1 /(i+j+1)=\int_{0}^{1} x^{i} x^{j} d x$, the matrix of $H$ is a Gramian and therefore positive. The operator $H^{2}$, being the product of two Hankel operators, belongs to $T$ (by (1)). Since $\mathbf{T}$ is a $C^{*}$-algebra, it contains the unique positive square root of each of its positive elements, and therefore, in particular, $\mathbf{T}$ contains the positive square root $H$ of $H^{2}$.

The Hilbert matrix is an illuminating example, but in an attempt to get new information about $\mathbf{E}$ and $\mathbf{T}$, it turned out to be a failure. It is, however, not a trivial failure. It belongs to $\mathbf{T}$, to be sure (and hence to $\mathbf{E}$ ), but not for the trivial reason; it doesn't belong to $\mathbf{K}$. Proof: if $f_{k}$ is the vector in $\mathbf{H}^{2}$ whose first $k$ coordinates are 
$1 / \sqrt{k}$ and all other coordinates are $0(k=1,2,3, \ldots)$, then $f_{k}$ is a unit vector and $f_{k} \rightarrow 0$ (weak). Since elementary estimates show that $\left(H f_{k}, f_{k}\right) \geqslant \frac{1}{2}$, the operator $H$ cannot be compact.

(9) Example. There are some near relatives of the Hilbert matrix that deserve examination. For each complex number $\alpha$ of absolute value 1 , let $H_{\alpha}$ be the operator with

$$
\left(\frac{\alpha^{i+j}}{i+j+1}\right)
$$

If $f_{k}$ is the vector whose initial coordinates are $1 /\left(\alpha^{j} \sqrt{k}\right)(j=0, \ldots, k-1)$ and all other coordinates are $0(k=1,2,3, \ldots)$ then, as before, $f_{k}$ tends to 0 weakly but $H_{\alpha} f_{k}$ does not tend to 0 strongly; the operator $H_{\alpha}$ is not compact. Does it belong to $\mathbf{E}$ ? The answer depends on $\alpha$. If $\alpha= \pm 1$, then $H_{\alpha} \in \mathrm{T}$; otherwise $H_{\alpha}$ doesn't even belong to E. Reason: straightforward computation shows that $U^{*} H_{\alpha} U-H_{\alpha}$ is a scalar multiple of $H_{\alpha}$ plus a compact operator. Consequence: $U^{*} H_{\alpha} U-H_{\alpha}$ is just as non-compact as $H_{\alpha}$.

(10) Example. The classically important Cesàro operator $C$ is defined by the matrix

$$
\left(\begin{array}{cccc}
1 & 0 & 0 & \\
\frac{1}{2} & \frac{1}{2} & 0 & \\
\frac{1}{3} & \frac{1}{3} & \frac{1}{3} & \\
& & & \ddots
\end{array}\right) .
$$

Is $C$ in $\mathbf{E}$ ? Yes, it is. Proof (straightforward computation): $U^{*} C U-C$ is a HilbertSchmidt operator.

Since $C$ is known to be hyponormal [1] and, in fact, subnormal [6], it follows that $C$ is not compact. Question: is $C$ in $\mathbf{T}_{0}$ ? Answer: no. Reason: if $T \in \mathbf{T}_{0}$, then $U^{*} T U-T$ has finite rank, but $U^{*} C U-C$ has a triangular matrix with all diagonal entries different from 0 , and therefore has infinite rank.

The preceding two comments are evidence, however weak, that $C$ does not belong to $\mathbf{T}$. There is a bit of evidence that $C$ does not belong to $\mathbf{T}$, namely that $C$ is an asymptotic Toeplitz operator. (In fact $\sigma(C)=0$, which shows incidentally that ker $\sigma \neq \mathbf{K}$. Cf. the proof of Corollary (6).) Is $C$ in $\mathbf{T}$ ? Nobody knows.

(11) Example. Which diagonal operators are in E? Which ones are in T? (In this context a diagonal operator is not just one that can be diagonalized, but one whose matrix with respect to the standard basis is diagonal.)

The answers are easy. If $T=\operatorname{diag}\left(\alpha_{0}, \alpha_{1}, \alpha_{2}, \ldots\right)$, then

$$
U^{*} T U-T=\operatorname{diag}\left(\alpha_{1}-\alpha_{0}, \alpha_{2}-\alpha_{1}, \alpha_{3}-\alpha_{2}, \ldots\right),
$$

and therefore a necessary and sufficient condition that $T \in \mathbf{E}$ is that $\alpha_{n+1}-\alpha_{n} \rightarrow 0$. Since $U^{* n} T U^{n}=\operatorname{diag}\left(\alpha_{n}, \alpha_{n+1}, \alpha_{n+2}, \ldots\right)$, it follows that $T$ is an asymptotic Toeplitz operator if and only if the sequence $\left\{\alpha_{n}\right\}$ is convergent. Note: if $\left\{\alpha_{n}\right\}$ is convergent, then $T \in T$. Proof: if $\alpha_{n} \rightarrow \alpha$, then

$$
T=\alpha+\operatorname{diag}\left(\alpha_{0}-\alpha, \alpha_{1}-\alpha, \alpha_{2}-\alpha, \ldots\right),
$$


and the diagonal summand is compact. Consequence: a diagonal operator is an asymptotic Toeplitz operator if and only if it belongs to the Toeplitz algebra. Conclusion: $T \in \mathrm{T}$ if and only if $\left\{\alpha_{n}\right\}$ is convergent.

Here at last is a source of decisive examples: to get an operator that is in $\mathbf{E}$ but not in $\mathbf{T}$, just construct a sequence that does not converge but whose first differences tend to 0 . That is easy, of course; form a sequence that oscillates between 0 and 1 more and more slowly. Concrete example:

$$
0, \frac{1}{2}, 0, \frac{1}{3}, \frac{2}{3}, 1, \frac{2}{3}, \frac{1}{3}, 0, \frac{1}{4}, \frac{2}{4}, \frac{3}{4}, 1, \frac{3}{4}, \frac{2}{4}, \frac{1}{4}, 0, \ldots
$$

(12) ExAMPLE. Is the adjoint of an asymptotic Toeplitz operator another one? No, not necessarily.

Consider an isometry $S$ defined on $\mathbf{H}^{2}$ by $S e_{n}=e_{2 n}, n=0,1,2, \ldots$, and write $T=S^{*}$. It follows that $T e_{2 n}=e_{n}$ and $T e_{2 n+1}=0, n=0,1,2 \ldots$ Consequence: for each $k$, the result of applying the "far southeast corner" $U^{* n} T U^{n}$ to $e_{k}$ results in the zero vector. Precisely, $U^{* n} T U^{n} e_{k}=0$ as soon as $n>k$. Conclusion: $U^{* n} T U^{n} \rightarrow 0$ (strong), so that $T$ is an asymptotic Toeplitz operator. The adjoint $T^{*}(=S$ ) is not. Reason: $U^{* n} S U^{n} e_{0}=U^{* n} S e_{n}=U^{* n} e_{2 n}=e_{n}$, and the sequence $\left\{e_{n}\right\}$ is not strongly convergent.

(13) ExAmple. Is the product of two asymptotic Toeplitz operators another one? No, not necessarily. An example can be obtained by modifying (11); the first such modification was suggested by $\mathrm{C}$. Foiaş.

Let $S_{k}$ be the square matrix of size $2 k$ defined as follows: all entries are 0 except the first $k$ in the last row, and they are equal to $1 / \sqrt{k}(k=1,2,3, \ldots)$. Let $S$ be the operator whose matrix is the direct sum of all the $S_{k}$ 's, and let $T$ be the adjoint of $S$.

Since $S e_{n} \rightarrow 0$ as $n \rightarrow 0$, it follows that $\left\|S U^{n} e_{k}\right\|=\left\|S e_{n+k}\right\| \rightarrow 0$ as $n \rightarrow \infty$, and hence that $\left\|U^{* n} S U^{n} e_{k}\right\| \rightarrow 0$ as $n \rightarrow \infty$ (for each $k$ ). This in turn implies that $S$ is an asymptotic Toeplitz operator (with $\sigma(S)=0$ ). So far the exact sizes of the boxes $S_{k}$ are irrelevant.

Consider next the matrix of the operator $T$. Since the only non-zero entry of $S_{1}^{*}$ is in the first row of $S_{1}^{*}$, it follows that both $T$ and $U^{*} T U$ begin with a column of 0 's, and, in fact, so does $U^{* n} T U^{n}$ whenever $n \geqslant 0$. Since the only non-zero entries of $S_{2}^{*}$ are in the first two rows of $S_{2}^{*}$, it follows that $U^{* 2} T U^{2}$ begins with two columns of 0 's, and so does $U^{* n} T U^{n}$ whenever $n \geqslant 2$. Inductively: $U^{* n} T U^{n}$ begins with $k$ columns of 0 's whenever $n \geqslant k(k+1)$. Consequence: $U^{* n} T U^{n} e_{k}=0$ as soon as $n \geqslant k(k+1)$ (usually sooner-the estimates are generous), so that $T$ is an asymptotic Toeplitz operator.

The product $S T$ is not an asymptotic Toeplitz operator. Reason: the diagonal entries of $S T$ are 0 most of the time, but 1 infinitely often. This implies that $U^{* n}(S T) U^{n} e_{0}=0$ most of the time but $e_{0}$ infinitely often, and, consequently, that the sequence $\left\{U^{* n}(S T) U^{n}\right\}$ is not strongly convergent.

(14) EXAMPLE. Typically a projection has a diagonal matrix with diagonal entries equal to 0 or 1 . Such a matrix can correspond to an asymptotic Toeplitz operator only if its rank is finite or cofinite. Are there any other asymptotic Toeplitz projections? 
Yes, there are. If $\mathbf{M}$ is a subspace of $\mathbf{H}^{2}$ invariant under $U$, then the projection from $\mathbf{H}^{2}$ onto $\mathbf{M}$ is in the Toeplitz algebra. Reason: by Beurling's theorem [5, Problem 125] there exists an inner function $\varphi$ such that $\mathbf{M}=\operatorname{ran} T_{\varphi}$; it follows that the projection in question is the product $T_{\varphi} T_{\varphi}^{*}$. (This observation is due to Sheldon Axler.)

There are asymptotic Toeplitz projections that do not seem to arise in the natural ways described in the preceding two paragraphs. Here is one. Let $T_{k}$ be the matrix of size $k$ all whose entries are equal to $\frac{1}{k}$, and form the matrix

$$
\left(\begin{array}{cccccc}
T_{1} & & & & & \\
& 0 & & & & \\
& & T_{2} & & & \\
& & & 0 & & \\
& & & & T_{3} & \\
& & & & & \ddots
\end{array}\right)
$$

that is the direct sum of the sequence obtained by interlacing a sequence of 0's (of size 1) with the $T_{k}$ 's. Clearly the operator $T$ with that matrix is a projection. Assertion: it is an asymptotic Toeplitz projection, with $\sigma(T)=0$. Reason: if the integer $n$ is such that the $n$th column of $T$ contains the first column of $T_{k}$, then $\left\|T e_{n}\right\|=\sqrt{k / k^{2}}=1 / \sqrt{k}$; for all larger $n$, the norm $\left\|T e_{n}\right\|$ is even smaller.

The reason the 0 's were inserted into $T$ was to make it easier to compute $U^{*} T U-T$. The computation has no virtues other than being easy to carry out. The result is that $U^{*} T U-T$ is block diagonal, and that the Hilbert-Schmidt norm of the $n$th block is of the order $1 / \sqrt{n}$. Conclusion: $U^{*} T U-T$ is not a Hilbert-Schmidt operator, but it is at least compact, and therefore $T \in \mathbf{E}$. Does $T$ belong to $\mathbf{T}$ or to $\mathbf{T}^{+}$? Nobody knows.

Questions. Two unsolved test problems have been posed already (see Examples (10) and (14)); each of them asks whether a certain operator belongs to T. That seems to be the crux of the matter in much of this subject. The important question is not "what is E?" but "what is T?". There is, after all, a way to decide whether or not an operator $T$ belongs to $\mathbf{E}$; just form $U^{*} T U-T$ and see whether it is compact. It's debatable whether this should be called an algorithm, but not even anything as good as that is known for T. The Hilbert matrix yields essentially the only non-trivial known example of an operator in $\mathbf{T}$; all others are either in $\mathbf{T}_{0}$, or compact, or both. Other non-trivial examples are easy enough to construct (e.g. non-trivial continuous functions of operators in $\mathbf{T}_{0}$ ), but the experts seem to agree nevertheless that the algebra $\mathbf{T}$ is far from well understood. The four questions below are special cases or reformulations of the general problem of characterizing the Toeplitz algebra.

The important classes discussed above are: the essential commutant E, the Toeplitz algebra $\mathbf{T}$, the Hankel algebra $\mathbf{T}^{+}$, and the set $\mathbf{T}^{\infty}$ of all asymptotic Toeplitz operators. The inclusion relations among them can be summarized by the Venn diagram, (15). 


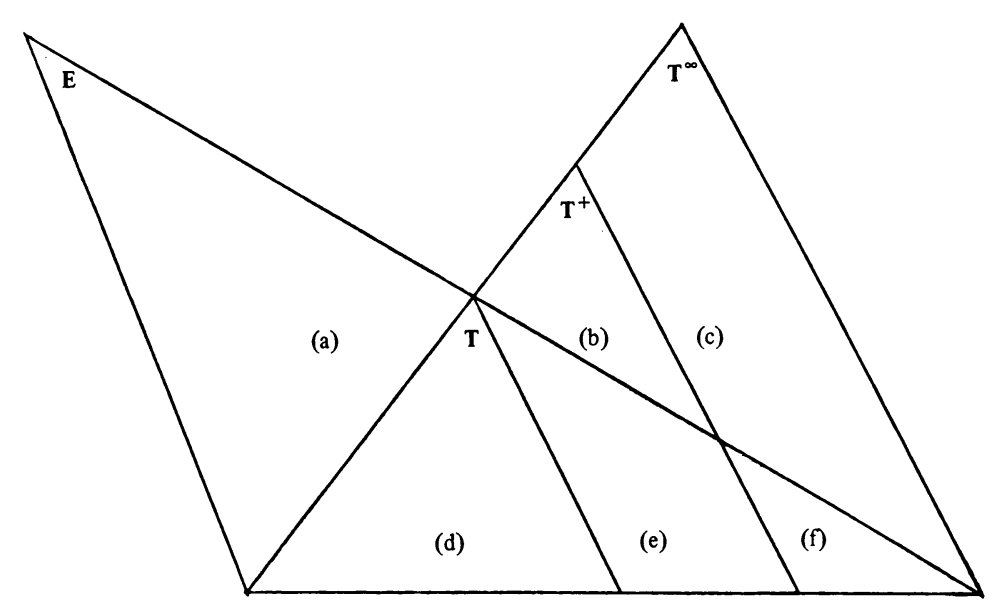

Operators corresponding to four of the indicated regions are known to exist; namely, (a) Example (11), (b) Example (9), (c) Example (12), and, for (d), any Toeplitz operator. Till now, however, no operators have been proved to belong to the classes (e) and (f).

(16) Question. Is there an operator in $\mathbf{E} \cap \mathbf{T}^{+}$that is not in $\mathbf{T}$ ?

(17) Question. Is there an operator in $\mathbf{E} \cap \mathbf{T}^{\infty}$ that is not in $\mathbf{T}^{+}$?

For each operator $T$ in the Toeplitz algebra, consider the difference $U^{*} T U-T$, and let $\mathbf{D}$ be the set of all such differences. Since $\mathbf{T} \subset \mathbf{E}$, it follows that $\mathbf{D} \subset \mathbf{K}$.

(18) Question. Which compact operators belong to $\mathbf{D}$ ?

The reason the question is interesting is that it is a reformulation of the question "which operators belong to T?". That is, the set D characterizes T. More clearly said, an operator $S$ belongs to $\mathbf{T}$ if and only if $U^{*} S U-S$ belongs to $\mathbf{D}$. Indeed, if $S \in \mathbf{T}$, then $U^{*} S U-S \in \mathbf{D}$ by definition. If, conversely, $U^{*} S U-S \in \mathbf{D}$, then, by definition, there exists an operator $T$ in $\mathrm{T}$ such that $U^{*} S U-S=U^{*} T U-T$. It follows that $U^{*}(S-T) U=S-T$, hence that $S-T$ is a Toeplitz operator, and hence that $S-T \in \mathbf{T}$. Conclusion: $S \in \mathbf{T}$.

Example (14) describes a projection in $\mathbf{T}^{\infty}$, and asks if it is in $\mathbf{T}$. It would be good to know the facts in the general case.

(19) Question. Which projections belong to T?

Problems frequently become more manageable, not less, if they are embedded in a suitable enlarged context. The last question to be raised here is vague; it isn't easy to formulate a crisp, yes-or-no subquestion, but it might give a hint to a suitably general context in which Toeplitz theory can be embedded.

Begin with the observation that Toeplitz operators are the solutions of the equation $U^{*} X U=X$. This suggests consideration of the mapping $\Gamma$ from operators to operators defined by

$$
\Gamma(X)=U^{*} X U .
$$

Toeplitz operators are the "eigenoperators" of $\Gamma$ corresponding to the eigenvalue 1 . Vague question: what is the spectral theory of $\Gamma$ ? What, in particular, can be said 
about eigenoperators $T$ (generalized Toeplitz operators), $U^{*} T U=\lambda T$, corresponding to eigenvalues $\lambda$ other than 1? What algebraic properties do they have, and what can be said about algebras generated by such operators?

\section{REFERENCES}

1. A. Brown, P. R. Halmos, and A. L. Shields, Cesàro operators, Acta Sci. Math. (Szeged) 26 (1965), 125-137.

2. K. R. Davidson, On operators commuting with Toeplitz operators modulo the compact operators, J. Funct. Anal. 24 (1977), 291-302.

3. R. G. Douglas, Banach algebra techniques in operator theory, Academic Press, New York, 1972.

4. __ Banach algebra techniques in the theory of Toeplitz operators, CBMS Regional Conf. Ser. in Math., no. 15, Amer. Math. Soc., Providence, R.I., 1973.

5. P. R. Halmos, A Hilbert space problem book, Springer-Verlag, New York, 1974.

6. T. L. Kriete, III and D. Trutt, The Cesàro operator in $l^{2}$ is subnormal, Amer. J. Math. 93 (1971), 215-225.

Instituto Venezolano de Investigaciones Cientificas, Apartado 1827, Caracas (101), VeneZUELA

DEPARTMENT OF MATHEMATICS, INDIANA UNIVERSITY, BloOMINGTON, INDIANA 47405 